\title{
The Oblateness of Large Hailstones
}

\author{
Keith A. Browning ${ }^{1}$ and Jelte G. D. Beimers \\ Royal Radar Establishment, Malvern, Worcestershire, England \\ (Manuscript received 28 July 1967)
}

\begin{abstract}
Ninety large oblate hailstones have been photographed to display thin sections containing their minor and major axes. The ratios of their minor to major axes have been determined throughout their growth from the shapes of the layered growth transitions. It has been found that the majority of hailstones became increasingly oblate throughout growth, but that a further increase in oblateness accompanied the melting of the hailstones during their descent to the ground.

Although even oblate hailstones are believed to tumble to some extent, they fall for most of the time with their minor axis vertical. Evidence is presented which suggests that during growth the proportion of unfrozen water is often greater at either end of the minor axis than at the sides of the hailstone. It is proposed that the increase in oblateness during growth is due to spongy ice on the upstream face being driven toward the sides of the hailstone, where it subsequently freezes, and that the further increase in oblateness during melting is a consequence of the more rapid melting of the relatively spongy ice remaining at the two ends of the minor axis. In extreme cases this process leads to the development of apple-shaped hailstones with pronounced indentations at one or both ends of the minor axis.
\end{abstract}

\section{Introduction}

Natural hailstones reaching the ground display a wide variety of shapes. Weickmann (1953), in summarizing reports of damaging hailstorms in Europe and North America during the previous 100 years, classified hailstones into three basic forms-conical, spheroidal and irregular. The relative frequencies with which these forms occurred were: conical $21 \%$, spheroidal $58 \%$, and irregular (including all stones with protuberances) $21 \%$. Of the spheroidal stones, $68 \%$ were markedly oblate; this corresponded to $40 \%$ of all hailstones.

More recently, Carte and Kidder (1966) have examined several thousand hailstones which fell in the Transvaal. Neglecting stones whose major axes were smaller than $1 \mathrm{~cm}$, they found that about one-quarter of the hailstones showed at least some degree of flatness, varying from just perceptible to disc shapes. The flattened hailstones, although basically oblate spheroids, included some apple-shaped stones in which indentations occurred at one or both ends of the minor axis.

Although, of course, very few hailstones ever conform precisely to any simple geometrical shape, there is little doubt that the oblate spheroid, albeit sometimes modified by a lobe structure or by apple-like indentations, is, in fact, one of the commonest shapes for hailstones reaching the ground. The question therefore arises as to how such a shape should arise: To what extent is the oblateness an intrinsic part of the growth

1 The raw data for this study were collected while this author was affiliated with the Air Force Cambridge Research Laboratories, Sudbury, Mass. itself and to what extent is it due to subsequent melting during the stone's descent to the ground? The present paper is an attempt to answer this question in the case of several samples of large hailstones collected in Oklahoma during May 1966.

\section{The hailstones}

Samples of large hailstones were collected from two locations after a storm in the Blackwell area on 15 May 1966, and from 18 locations after storms in the El Reno and Oklahoma City areas on 20 May 1966.

Some of the samples were obtained by special mobile hail collecting teams, but most were collected from householders who had stored the hailstones in their refrigerators. Altogether, 134 large hailstones were collected and sectioned, but of these 44 were discarded either because they grew too irregularly to be described in terms of a basically spheroidal shape or because there was evidence that melting had occurred after the hailstones had reached the ground. The remaining 90 hailstones that were analyzed in detail had major axes varying between 2 and $6 \mathrm{~cm}$ in length. Of these the majority $(85 \%)$ were between 3 and $5 \mathrm{~cm}$.

Outside dimensions of the 90 analyzed hailstones were first measured in terms of the three axes of an ellipsoid- $2 a, 2 b$ and $2 c$, where $a>b>c$. The ratio $b / a$ was found to be equal to or greater than 0.9 for $61 \%$ of the stones and was less than 0.7 for only $5 \%$ of them. On the other hand, the ratio $c / a$ was greater than or equal to 0.9 for only $7 \%$ of the stones while it was less than 0.7 for $50 \%$. Thus, although the shape of the stones 

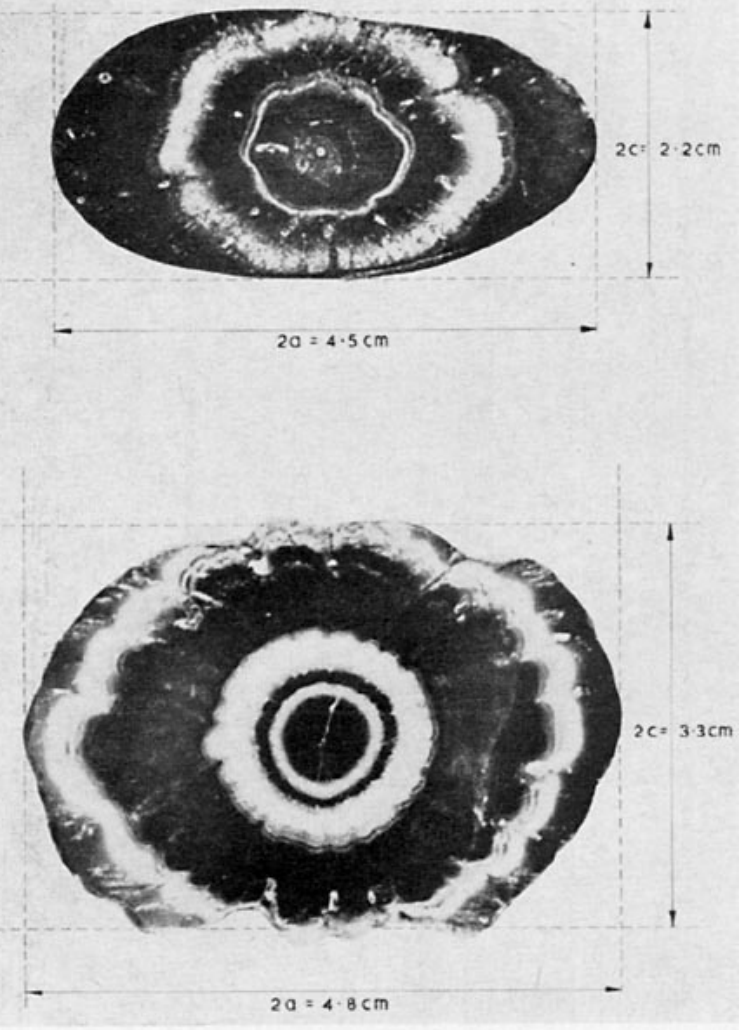

FIG. 1a and 2a. Photographs of thin sections through two large hailstones taken by transmitted light such that regions of transparent ice appear black and regions of cloudy ice containing many air bubbles appear white. The sections were cut so as to pass close to the growth centers and to contain the longest $(2 a)$ and shortest (2c) axes. (Photographs by J. Burnham, Royal Aircraft Establishment, Bedford.)

could be approximated more closely by ellipsoids, the two larger axes were usually rather similar in length and both were commonly significantly larger than the third. Hence, it is convenient to regard most of these stones as being more or less oblate spheroids.

\section{Analytical technique}

The hailstones were sectioned using a simple band saw, 1-2 mm thick sections being obtained close to their growth centers so as to contain their major and minor axes. These sections were mounted on glass slides and photographed by plain transmitted light. Two examples are shown in Figs. 1a and 2a. The analysis is concerned with the ratio $c / a$, which we will call $\alpha$. Values of $\alpha$ have been derived at different stages of growth from the shape of transitions between layers of transparent and cloudy ice. By using a set of elliptical overlays constructed with eccentricities at 0.05 intervals, it was possible to estimate a best-fitting $\alpha$ to an accuracy of \pm 0.025 . Sometimes, as in Fig. $2 \mathrm{a}$, the outermost transitions were incomplete due to sub- sequent melting, in which case $\alpha$ has had to be determined on the basis of partly extrapolated growth transitions.

The outside surface of the hailstones was almost always modified by melting; often the melting took place preferentially at the two ends of the minor axis, thereby producing somewhat flattened non-elliptical sections, as in Fig. 2a. In these circumstances a more representative value of $\alpha$ for the outside surface of the photographed section was derived using the values of $a$ and $c$ obtained as shown in Figs. 1a and 2a.

The trends of $\alpha$ as a function of $a$ during the growth and subsequent melting of the hailstones depicted in Figs. 1a and 2a are shown graphically in Figs. $1 \mathrm{~b}$ and 2b. Similar plots have been obtained for all 90 analyzed hailstones. Growth transitions are indicated by filled circles or crosses according to whether they were from transparent to cloudy ice or in the opposite sense. Grow th transitions that were incomplete due to melting are denoted by bracketed points. The value of $\alpha$ for the outside surface of the hailstone is denoted by a large open circle.

In order to provide a measure of the mean rate of change of $\alpha$ during growth, a straight line has been fitted to all points, with the exception of the one corresponding to the outside surface, using the method of least squares. Generally, as in the case of Figs. 1 and 2 , the rate of change of $\alpha$ with hailstone size remained in the same sense (with only minor departures) throughout growth, so that the straight line was a good description of the entire growth phase.

The effect of melting has been obtained in terms of the difference $\Delta \alpha_{m}$ between the value of $\alpha$ for the outside surface and the extrapolated value of $\alpha$ at the corresponding value of $a$ based upon the best-fit straight line. That is to say, the effect of melting has been calculated on the assumption that the rate of change of $\alpha$ during the final phases of growth remained the same as the mean value during the earlier growth phase. (There were a few hailstones for which there was slight evidence that the rate of decrease of $\alpha$ diminished as the stone neared its maximum size, and in these cases the effect of melting in further decreasing $\alpha$ will have been underestimated; however, since there appeared to be no satisfactory way of accounting for these cases objectively, they have not been corrected for, and this therefore remains as a minor source of error).

\section{The results}

Fig. 3 shows the frequency distribution of the quantity $(d \alpha / d a)_{g}$ corresponding to the rate of change of oblateness with increasing semi-major axis during growth. Although there is a wide spread of values, most hailstones showed a tendency to become more oblate during growth; of the 90 hailstones only 14 showed a tendency to become less oblate. The mean value of 

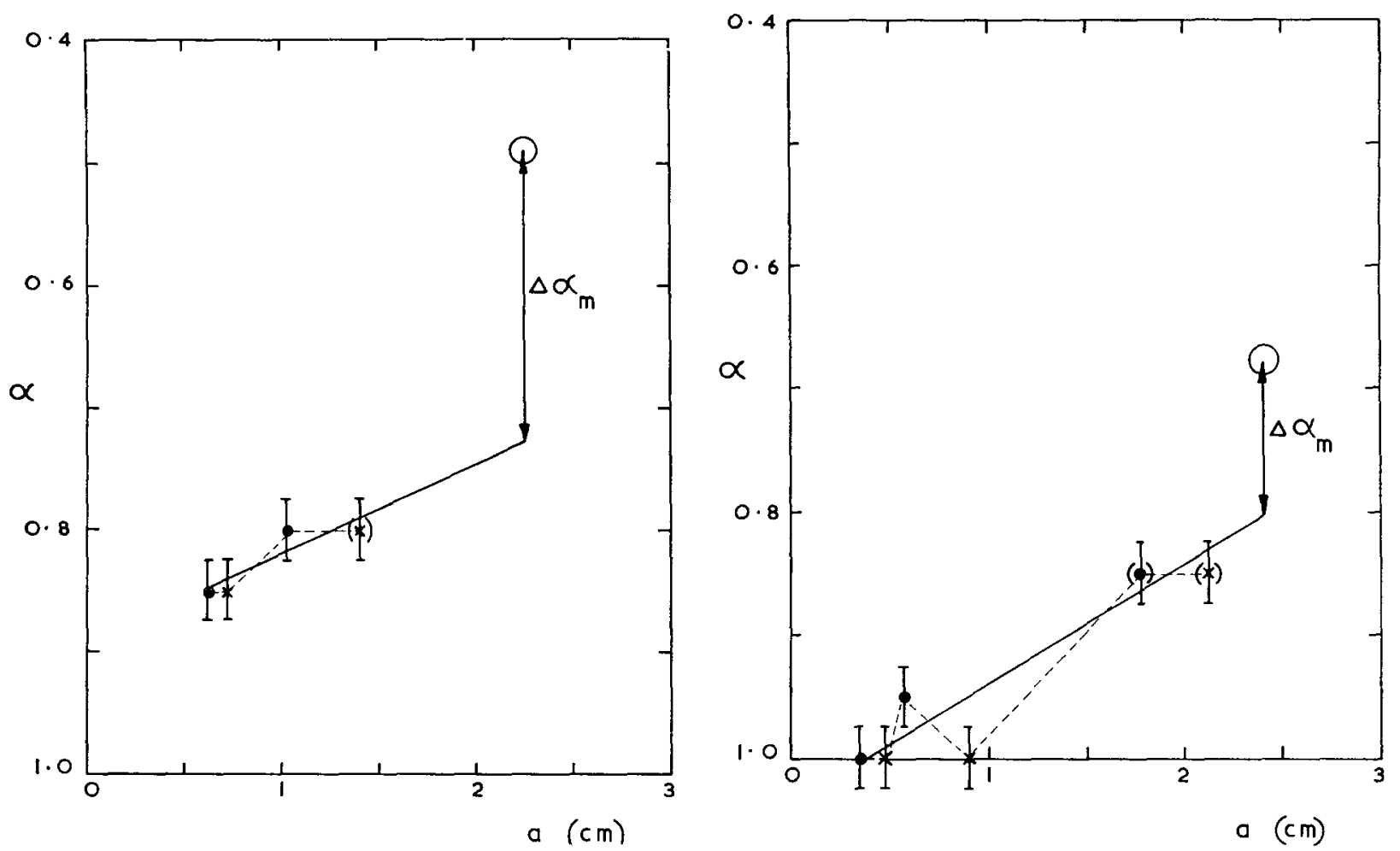

FIGs. $1 \mathrm{~b}$ and $2 \mathrm{~b}$. The ratio of the minor to major axes $\alpha$ plotted as a function of the semi-major axis $a$ for the hailstone sections portrayed in Figs. 1a and 2a. Filled circles and crosses, respectively, have been plotted on the basis of transitions from transparent to cloudy ice, and vice versa. Bracketed values are based upon growth transitions that were incomplete due to melting. The value of $\alpha$ for the outside surface of the hailstone, obtained using the dimensions indicated in Figs. 1a and 2a, are plotted as large open circles. The value $\Delta \alpha_{m}$ corresponds to the change of $\alpha$ attributed to melting. The solid line is a best-fit straight line through all points except that for the outside surface; its slope gives a measure of $(d \alpha / d a)_{a}$, the rate of change of $\alpha$ during growth.

$(d \alpha / d a)_{\text {o was }}-0.09 \mathrm{~cm}^{-1}$. Since the mean value of $a$ for the outside surface of the 90 hailstones was $2.0 \mathrm{~cm}$, this corresponds to an estimated average total change of $\alpha$ during growth of -0.18 .

Fig. 4 shows the frequency distribution of the quantity $\Delta \alpha_{m}$, corresponding to the further change of oblateness attributable to melting. Again there is a wide spread of values, with a majority of hailstones showing a tendency to become more oblate during melting; of the 90 hailstones only 11 showed a tendency

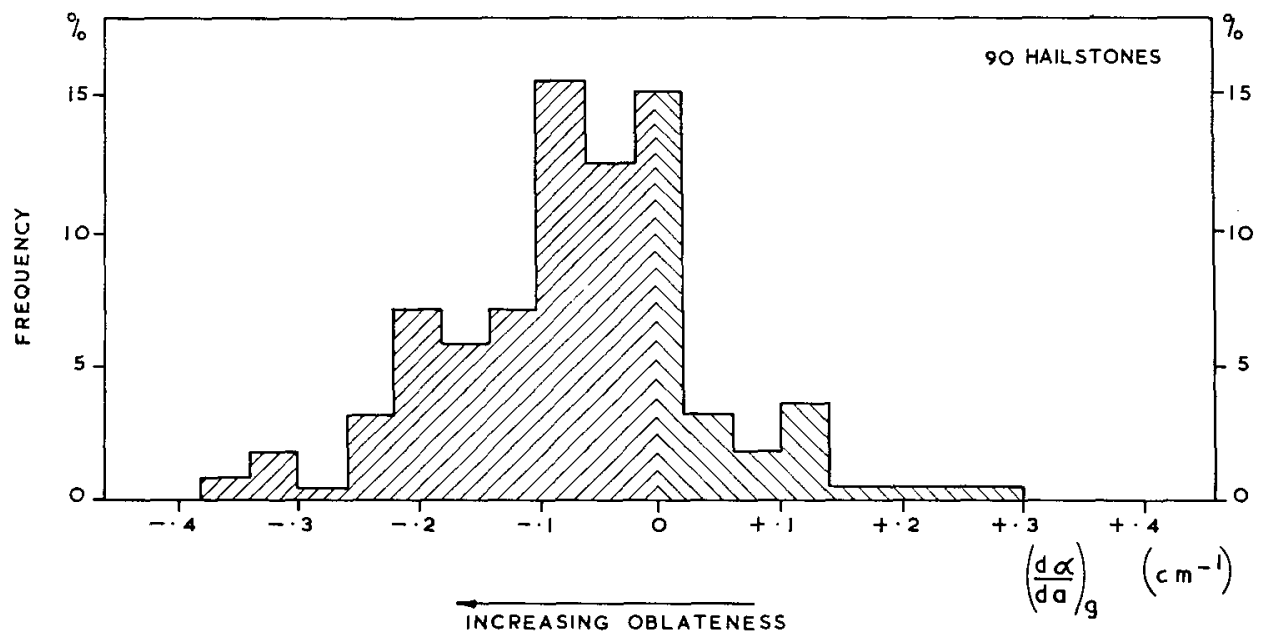

FIG. 3. Frequency distribution of $(d \alpha / d a)_{0}$ for 90 hailstones plotted in increments of $0.04 \mathrm{~cm}^{-1}$. Notice the preponderance of negative values, indicating an increase of oblateness during growth. 


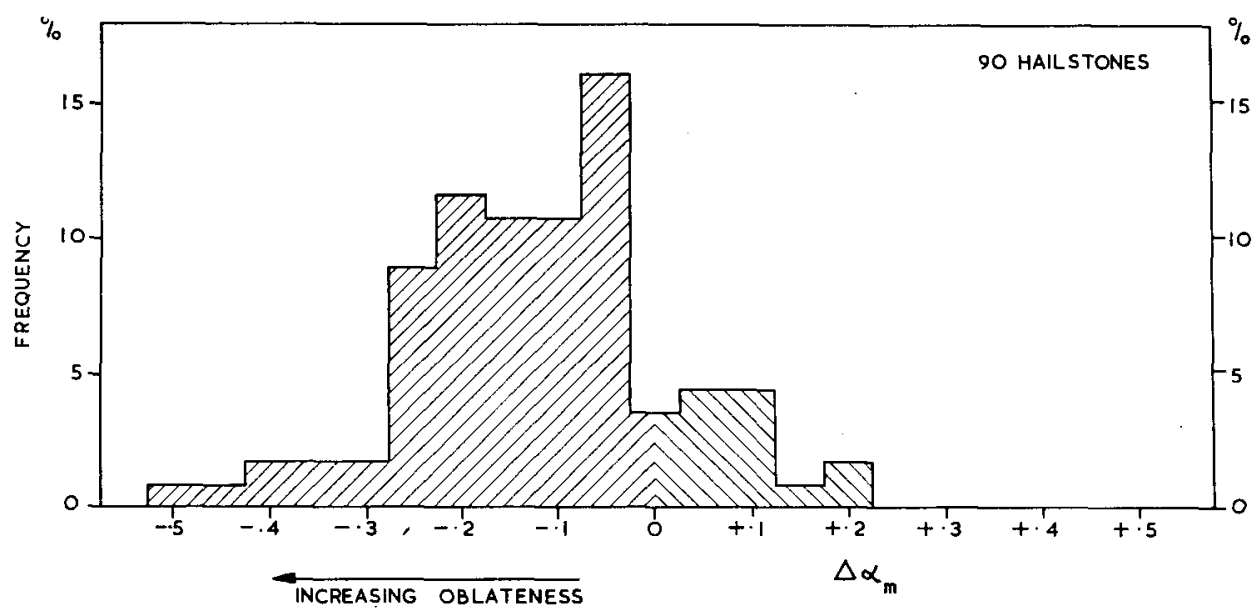

FIG. 4. Frequency distribution of $\Delta \alpha_{m}$ for 90 hailstones plotted in increments of 0.05 . Notice the preponderance of negative values, indicating an increase of oblateness due to melting.

to become less oblate. The mean change of $\alpha$ due to melting was -0.13 .

Fig. 5 shows the dependence of $\alpha_{s}$, for the outside surface of the hailstones, upon $\alpha_{1}$, corresponding to an early stage in the growth when $2 a=1 \mathrm{~cm}$. Evidently, a large proportion of the hailstones were almost spherical at first, ${ }^{2}$ the median value of $\alpha_{1}$ being 0.94 , corresponding to the vertical dashed line in Fig. 5. (The fact that

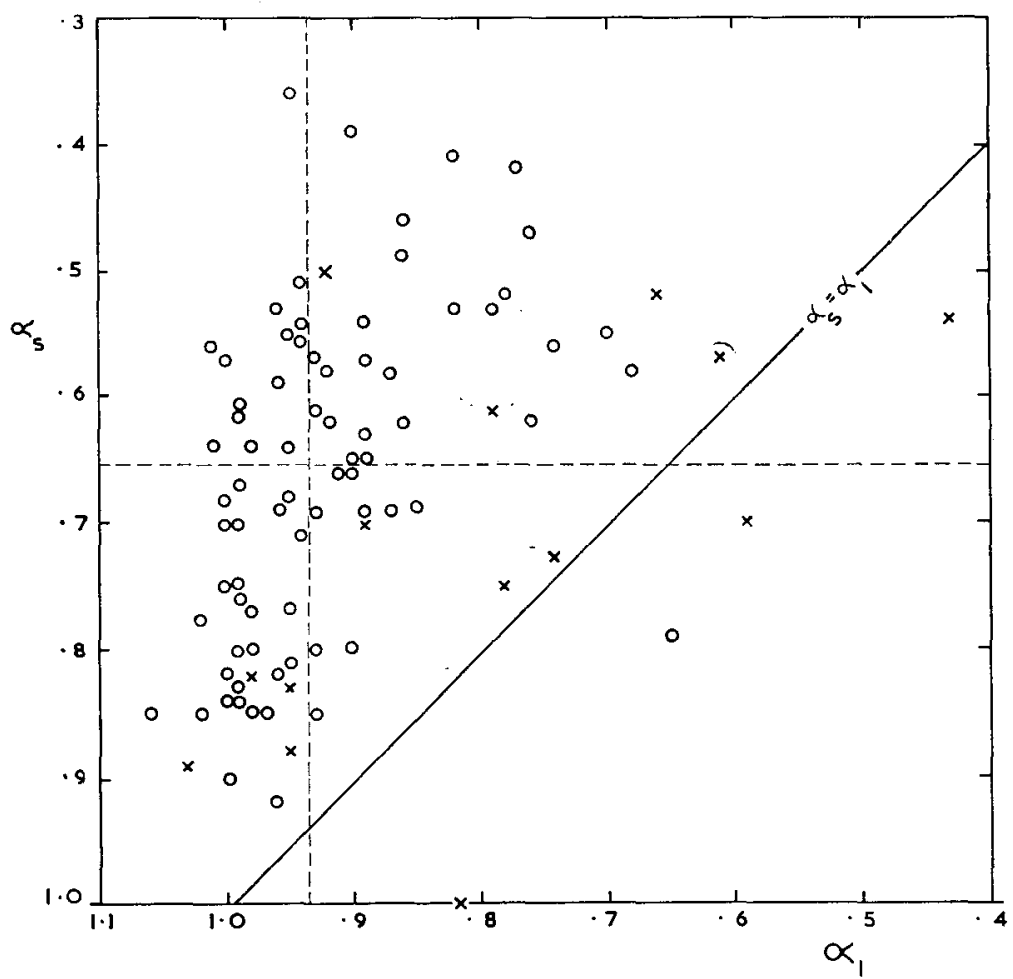

FIG. 5. The ratio of minor to major axes at the hailstone surface $\alpha_{s}$ plotted as a function of the same ratio when the major axis was $1 \mathrm{~cm}\left(\alpha_{1}\right)$ for 90 hailstones. Crosses refer to hailstones which became less oblate during growth; all other hailstones are represented by circles. Median values of $\alpha_{s}$ and $\alpha_{1}$ are indicated by the horizontal and vertical dashed lines. Notice that the majority of the hailstones were only very slightly oblate when they were $1 \mathrm{~cm}$ in diameter but that most of them became markedly oblate by the combined effects of growth and melting.

${ }^{2} \mathrm{~A}$ few hailstones with large graupel particles as embryos were excluded because of the difficulty of fitting ellipses to the photographed sections. 
$\alpha_{1}$ sometimes slightly exceeded unity is of little significance and is due merely to the minor and major axes being defined with respect to the shape of the fullygrown hailstone). Owing to the combined effects of growth and melting, the median value of $\alpha_{s}$, on the other hand, was only $0.66, \alpha_{s}$ exceeding $\alpha_{1}$ for only 4 of the 90 hailstones. Crosses plotted in Fig. 5 correspond to the 14 hailstones that became increasingly less oblate as they grew, but except for 3 of these, the change in oblateness due to melting predominated so as to cause $\alpha_{s}$ to be smaller than $\alpha_{1}$.

\section{Interpretation of the results}

a. Change of oblateness during growth. Once a hailstone gets beyond its initial conical stage it tends to fall with its largest cross section facing the airstream (List, 1959). Further growth on the side facing the airstream will then act relatively to enhance the crosssectional area with respect to an airflow oriented at right angles to the initial direction and the stone will develop a tendency to turn through $90^{\circ}$. If the hailstone surface were not being molded aerodynamically during growth, this process would occur repeatedly. The resulting tumbling would then be expected to give rise to growth layers of fairly uniform thickness and to virtually spherical, although perhaps convoluted, hailstones (Browning, 1966). Fig. 3 shows that the hailstones analyzed in the present paper generally did not follow this pattern; instead of $\alpha$ remaining close to unity during growth, negative values of $(d \alpha / d a)_{g}$ predominated. Although growth occurred on all sides of the hailstones for most of the time, thereby indicating that the stones probably were tumbling to some extent, the growth tended to occur fastest near the ends of the major axes, i.e., on those parts of the hailstones which can be expected to have spent most time oriented at right angles to the airflow. This suggests that droplets were not remaining on the hailstone surface in the positions where they were accreted but, rather, that the accreted material was being molded aerodynamically.

Aerodynamic molding of growing hailstones has been simulated in the laboratory by Mossop and Kidder (1962). Because they used hailstone models suspended with only one degree of freedom they obtained rather unrealistic rim-like shapes. Nevertheless, they were able to reproduce the broad features of enhanced growth in directions perpendicular to the airflow. They found that this kind of molding occurred only under conditions of wet growth, when the ice was growing spongily. List (1965) has interpreted this to imply a relatively low flow resistivity of the spongy ice framework, which fails to counteract the pressure drop between the stagnation point and the outer periphery of the hailstone cross section facing the airflow.

About $70 \%$ of the hailstones in the present study had clearly defined lobe structures and, according to Browning (1966), lobes themselves contain only rela- tively small amounts of unfrozen water. At first sight this appears to contradict List's interpretation; however, in the models proposed by Browning (1966) and independently by Sarrica (1965), the interstices between the lobes are liable to become filled with a high proportion of unfrozen water and this may be expected to flow toward the sides of the hailstone as List has suggested. In circumstances of very wet growth, as we shall show later, the spongy interstices become large compared with the lobes, and the lobes themselves then tend to become distorted by the airflow.

b. Change of oblateness during melting. The fact, demonstrated in Fig. 4, that melting usually further increased the oblateness of hailstones is in accord with the experiments of Blanchard (1957), Mossop and Kidder (1962), and Macklin (1963, 1964). The presence of a few hailstones in Fig. 4 which showed the opposite trend (i.e., positive $\Delta \alpha_{m}$ ) is probably due to the limitations of the analysis, because the value of $\alpha$ just before melting could only be estimated by extrapolation of the earlier trend.

There are several possible explanations of this effect. Blanchard (1957), for example, found that melt water forms a belt around the spheroid which insulates the ice beneath so that melting takes place preferentially at its top and bottom. Macklin (1964), however, points out that, at least in the case of a smooth sphere, the heat transfer is greater at the front than at the sides even in the absence of a belt of melt-water and that it increases again on the downstream side owing to turbulence in the wake. In the following section we shall present evidence for a third explanation-one which appears to contradict the explanation proposed by Macklin.

\section{A mechanism for the production of oblate hailstones}

A feature clearly distinguished in about half of the oblate hailstones in the present study was that, within layers of transparent ice, streams of fairly large air bubbles indicative of spongy ice (Browning, 1966; Bailey and Macklin, 1967) occur more commonly near the top and bottom of the hailstone (i.e., at either end of the minor axis) than near its sides. Fig. 6a illustrates this point particularly clearly; it shows a layer of transparent ice which, although virtually free of air bubbles near the two sides, contains quasi-radial streams of fairly large bubbles elsewhere. The presence of spongy ice near the top and bottom of the hailstone, which this indicates, provides a new explanation for the relatively fast melting that tends to occur in these locations. It also accounts for the occasional extreme melting that leads to apple-shaped hailstones such as the one protrayed in Fig. 7. In this example again note the increase in the abundance of streams of large air bubbles in the outer transparent ice layer toward 


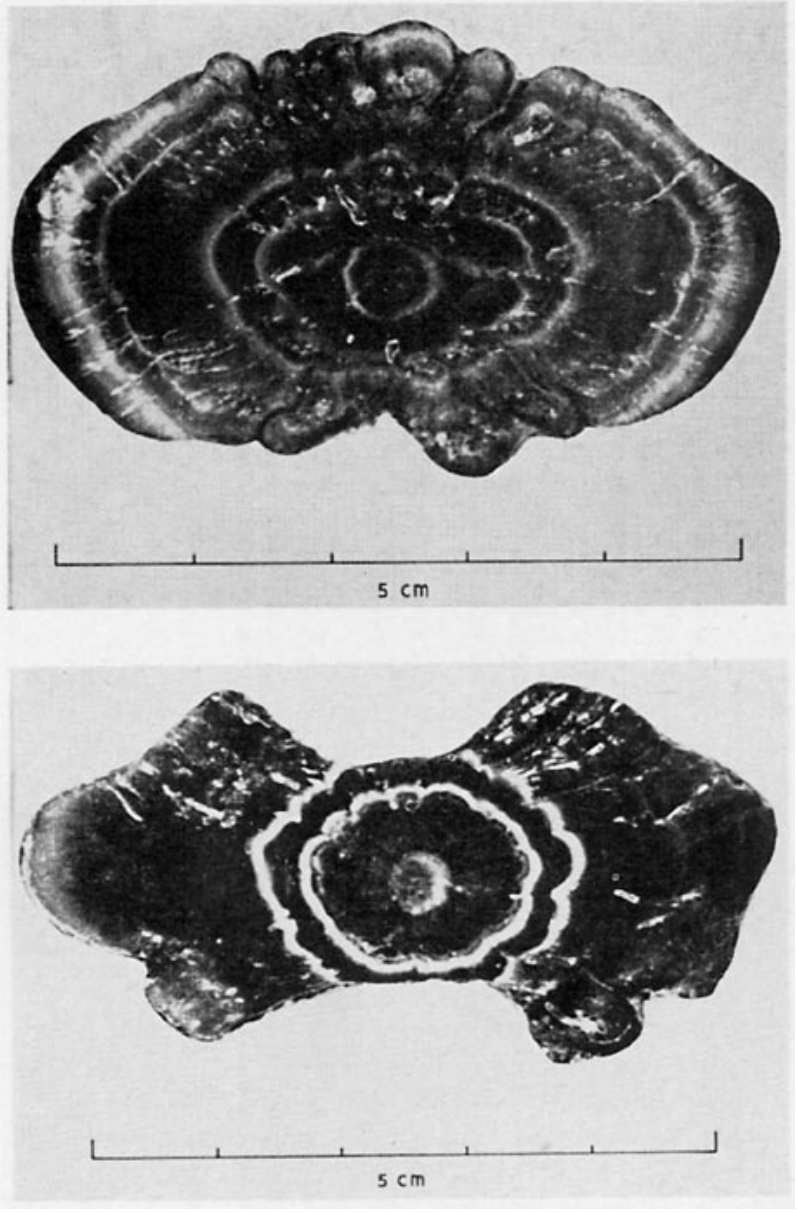

Figs. 6a and 7. Photographs of thin sections through two large hailstones taken by transmitted light such that regions of transparent ice appear black and regions of cloudy ice containing many air bubbles appear white. The sections were cut so as to pass close to the growth centers and to contain the longest and shortest axes. (Photographs by J. Burnham, Royal Aircraft Establishment, Bedford.)

the top and bottom of the hailstone close to the regions where the pronounced melting has occurred.

Our suggestion that oblate hailstones tend to contain a larger proportion of unfrozen water toward their top and bottom also provides a clearer picture of what causes the stones to grow oblately in the first place. Fig. 6b is a simplified sketch of the photograph in Fig. 6a which draws attention to the salient features. The layered structure of the hailstone is depicted schematically by stippling and the streams of large air bubbles, which can occasionally be identified as lying along the interstices of ill-defined lobes, are drawn as solid lines. In other hailstones which grow more nearly spherically, the lobes and the streams of large bubbles tend to be oriented radially. In Fig. $6 \mathrm{~b}$, however, the the lines are distorted in a manner which suggests that the accreted material has been driven toward the sides of the hailstone, presumably in a manner similar to that described by List (1965).

\section{Concluding remarks}

It appears that there is a tendency for hailstones to grow more spongily at either end-of their shortest axis. Material accreted in these locations (when either one end or the other is facing the airstream) is to some extend driven toward the side of the hailstone, where it subsequently freezes, and so the hailstone becomes increasingly oblate as it grows. When the stone melts during its final descent to the ground, it does so fastest at the two ends of its minor axis where the proportion of unfrozen water is already relatively high, and so its oblateness is further increased. This explanation implies that the heat transfer, instead of being greater at the two ends of the minor axis, as Macklin (1964) suggests, is in fact more efficient around the sides of the hailstones.

In order to simulate this mechanism realistically in the laboratory, it will be necessary to grow hailstones in free suspension in a vertical hail tunnel, as Bailey and Macklin (1967) have done. Because of the small working cross section of their tunnel, however, strong horizontal gradients of vertical velocity occurred which tended to prevent hailstones from maintaining the kind of semi-stable orientation that is required to produce oblate hailstones resembling those found in nature. Needless to say, it will not be an easy task to design a tunnel in which the profile of vertical velocity is sufficiently uniform in the horizontal to permit hailstones to grow oblately while remaining freely supported.

Acknowledgments. The authors are indebted to Dr. E. Kessler, Director of the National Severe Storms Laboratory, and his staff for their cooperation. We are also grateful to students of Oklahoma University, to Drs. J. Hallett, D. Johnson and E. Kuhns (Imperial College), and to Dr. W. T. Roach and Messrs. T. W. Harrold and G. Lowe (Meteorological Office) for their combined efforts in collecting the hailstones. We are particularly grateful to Mr. J. Burnham (Royal Aircraft

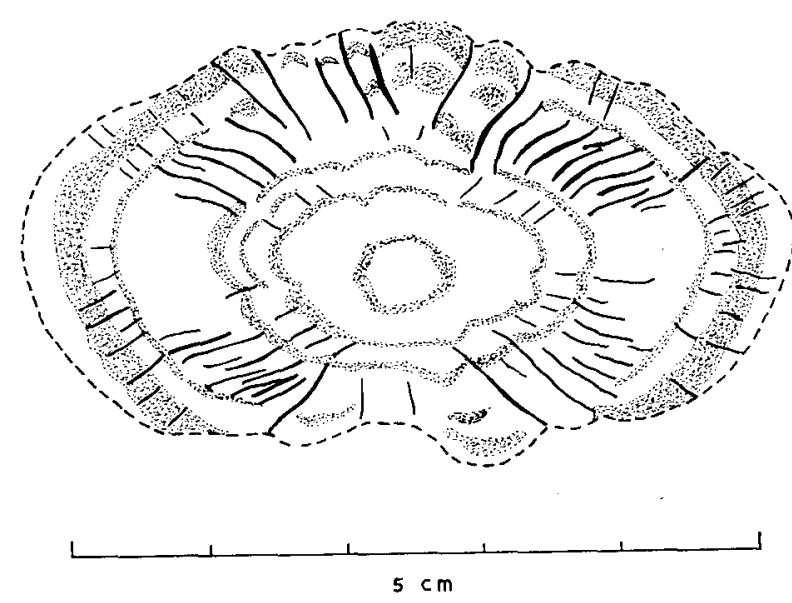

FIG. 6b. Sketch of the hailstone section in Fig. 6a showing the salient features. Layers of cloudy ice are depicted schematically as stippled regions, and streams of large air bubbles are represented by solid lines. 
Establishment, Bedford, England) for photographing the hailstone sections. This article has been contributed with the permission of the Director General of the Meteorological Office, the Director of the Royal Radar Establishment and the Copyright Controller H.M.S.O.

\section{REFERENCES}

Blanchard, D. C., 1957: The supercooling, freezing, and melting of giant waterdrops at terminal velocity in air. Artificial Stimulation of Rain. New York, Pergamon Press, 233-249.

Bailey, I. H., and W. C. Macklin, 1967: The surface configuration and internal structure of artificial hailstones. Quart. J. Roy. Meteor. Soc. (in press).

Browning, K. A. 1966: The lobe structure of giant hailstones. Quart. J. Roy. Meteor. Soc., 92, 1-14.
Carte, A. E., and R. E. Kidder, 1966: Transvaal hailstones. Quart. J. Roy. Meteor. Soc., 92, 382-391.

List, R., 1959: Zur Aerodynamik von Hagelkörnern. Z. Angero. Malh. Phys., 10, 143-159.

- 1965: The mechanism of hailstone formation. Proc. International Conf. Cloud Physics, Tokyo and Sapporo, 481-491.

Macklin, W. C., 1963: Heat transfer from hailstones. Quart. J. Roy. Meteor. Soc., 89, 360-369.

- 1964 : Factors affecting the heat transfer from hailstones. Quart. J. Roy. Meteor. Soc., 90, 84-90.

Mossop, S. C., and R. E. Kidder, 1962: Artificial hailstones. Bull. Obs. Puy de Dome, 2, 65-80.

Sarrica, O., 1965: Observational results on hail formation and structure. Ric. Sci., 35, (II-A), 345-359.

Weickmann, H., 1953: Observational data on the formation of precipitation in cumulonimbus clouds. Thunderstorm Flectricity, University of Chicago Press, 66-138. 